\title{
Assessment of Myocardial Ischemia through High Frequency Energy Estimation over the Time-Frequency Plane using Wavelets
}

\author{
N Castro ${ }^{1}, \mathrm{P}_{\text {Gomis }}{ }^{1,2}$, G Wagner ${ }^{3}$ \\ ${ }^{1}$ Universidad Simón Bolívar, Caracas, Venezuela \\ ${ }^{2}$ CREB, Universitat Politècnica de Catalunya, Barcelona, España \\ ${ }^{3}$ Duke University Medical Center, Durham, North Carolina, USA
}

\begin{abstract}
This study describes total energy changes over eight regions of the QRS time-frequency (TF) plane, during percutaneous transluminal coronary angioplasty (PTCA). The study population consisted of 43 patients undergoing PTCA, with no previous myocardial infarction history: 25 in the right coronary (RCA), 10 in left anterior descending (LAD) and 8 in left circumflex (LCX) arteries. Blockwise and exponential signal averaging technics were used at pre-processing stage; continuous Morlet wavelet transform and total energy calculation were applied. Significant changes were found in the HF regions $(p<0.05)$ in patients assessed at the end of the PTCA (80-250Hz/second QRS half) compared with their values previous to inflation. Total energy over specific time-frequency regions, assessing high frequency using a frequency band from 80 to $250 \mathrm{~Hz}$, could be used as a marker for ischemic events during depolarization. These results suggest that leads II and aVF are more sensitive to such changes.
\end{abstract}

\section{Introduction}

During ischemic events myocardial cells must abandon electric and mechanic coupling and stay into rest in order to survive [1]. This may result, mainly, in a deviation of final wave QRS amplitudes in the same direction of ST segment [2], more probably as a consequence of slow myocardium electric activation. Reversible ischemia was generally measured only during the repolarization process through ST segment deviation. Recent studies suggest that ischemic events can produce changes in highfrequency components of the QRS complex [3, 4]. Because of its multi-resolution signal decomposition, wavelet analysis and specifically complex Morlet wavelet has been successfully used to characterize qualitatively patients prone to ventricular tachycardia (VT) $[5,6]$ and short lasting events with the surface ECG before and after angioplasty [7]. Different studies have reported high frequency energy changes (HFEC) during acute coronary occlusion. Abboud et al. and Mor Avi et al [3, 8] studied this phenomenon over human and animal models respectively; Petterson et al. [4] could find HFEC even without ST deviations and obtained better sensitivity and specificity than the ST criteria. These studies of HFEC have used the $150-250 \mathrm{~Hz}$ bandwidth, however, others research have included the bands of $80-300 \mathrm{~Hz}$ [9], 20$30 \mathrm{~Hz}$, and $16-200 \mathrm{~Hz}$ [7], which shows that there is not a specific time-frequency region to evaluate HFEC. The purpose of this paper is searching high frequency bands within the QRS related with ischemia produced during percutaneous transluminal coronary angioplasty (PTCA). We focused on eight time-frequency regions over the QRS where ischemic events could produce HFEC caused by coronary occlusion. Rectangle regions were used in the time-frequency $(t-f)$ plane, but our aim is finding a "fingerprint" of HFEC over the t-f plane.

\section{Materials and methods}

\subsection{Database}

This study used the Staff-3 database, obtained at Charleston Area Medical Center. It is formed by ECG registers before, during and after PTCA, which constitute an appropriate model to study ischemia. Signals were acquired with equipment Siemens-Elena (Solna-Sweden) at $1 \mathrm{kHz}, 0.6 \mu \mathrm{V}$ resolution and stored on hard disk for posterior analysis. Standard leads I, II, III and precordials V1, V2, V3, V4, V5 and V6 were digitized. The whole database include 108 patients, a first selection considered 57 patients among those without previous myocardial infarction history according with the ECG QRS scoring system of Selvester et al. [10]. 27 patients had occlusion of right coronary artery (RCA), 19 of left anterior descending (LAD) and 11 in left circumflex (LCX) arteries. A second selection was necessary due to noise bursts (see fig. 1) mainly in some PTCA registers. Finally a smaller study group of 39 patients were analyzed: 23 RCA, 8 LAD and 8 LCX (25 males, 14 females: $61 \pm 11$ 
years). Each patient has a 5 minute pre-PTCA ECG register in supine position at catheterism lab and a PTCA ECG register with a duration occlusion (balloon inflation) raged from 1' 30 '' to 7' 17 ', (mean 4' 28 '') and several minutes of post-inflation time.

\subsection{Pre-processing}

\subsubsection{Signal averaging}

A low noise level was required for posterior analysis of low amplitude high-frequency signals, thus a signal averaging process was carried out in two ways: blockwise averaging was applied to 5 minutes pre-PTCA registers and an exponentially updated beat was used for PTCA registers, for preserving morphologic changes during inflation. The weight defining this exponential updated was made noise dependent, then a slower update was used during noise bursts. Averaging process used 10 minutes trend samples for updating of pre and PTCA registers, a 0.94 cross correlation coefficient was used for alignment purposes. Each trend sample was visually checked for updating slowing at regions of interest, like final inflation beat; this leaded to the second selection study group as previously stated. We assumed low-pass filtering averaging effect as an unavoidable error source coming from misalignment, since crude beats had unacceptable noise levels.

\subsubsection{Beat selection}

The beat selection for processing stage calculations were done from averaging process trend series. Final inflation beat was selected as the beat where ischemic high frequency changes had strongly been manifested. In each patient, the control beat for comparison purposes was chosen either from pre-PTCA registers or from PTCA registers in the time previous to occlusion, averaging the noise level of V2, V4, I and II leads and chosing the control beat with similar noise level of the PTCA beat (see fig. 1). Leads with noise level higher than $75 \mu \mathrm{V}$ at the end of the occlusion were rejected.

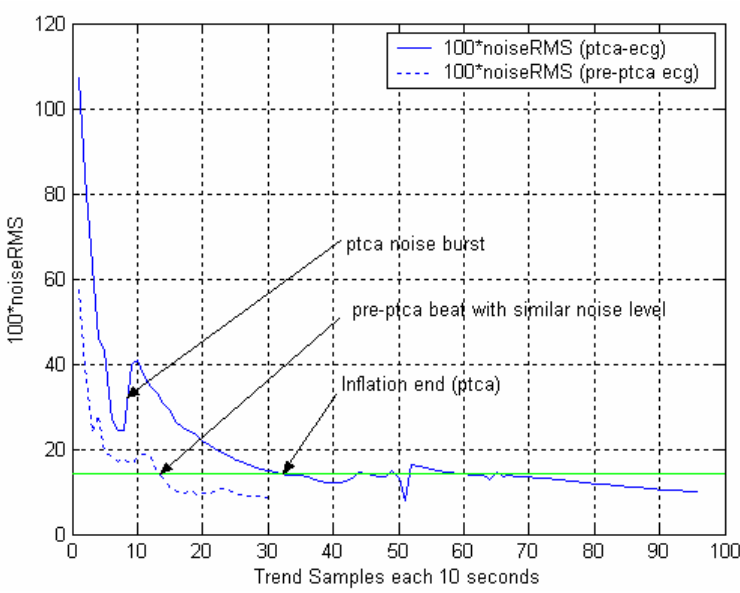

Figure 1. Exponential noise decay and similar noise beat selection: patient number 10 (Staff-3 database) $[\mu \mathrm{V}]$.

\subsubsection{Time-frequency regions}

The time-frequency regions of interest were define dividing the QRS into two halves, using the frequency bands: R1, R3: (16-80 Hz); R2, R4: (80-150Hz); R5, R6:(150-250Hz) and R7, R8: (80-250Hz), corresponding $\mathrm{R} 1, \mathrm{R} 2, \mathrm{R} 5$ and R7 to the first half of the QRS and R3, $\mathrm{R} 4, \mathrm{R} 6$ and $\mathrm{R} 8$ to the second half as shown in fig. 2

\subsubsection{Continuous wavelet transform}

The continuous wavelet transform (CWT) defined in (1), was used to compute the complex coefficients to calculate total energy by each region as define previously over all leads and patients.

$$
W(\tau, a)=<s \mid g_{\tau, a}>=\frac{1}{\sqrt{a}} \int s(t) g_{m}^{*}\left(\frac{t-\tau}{a}\right) d t
$$




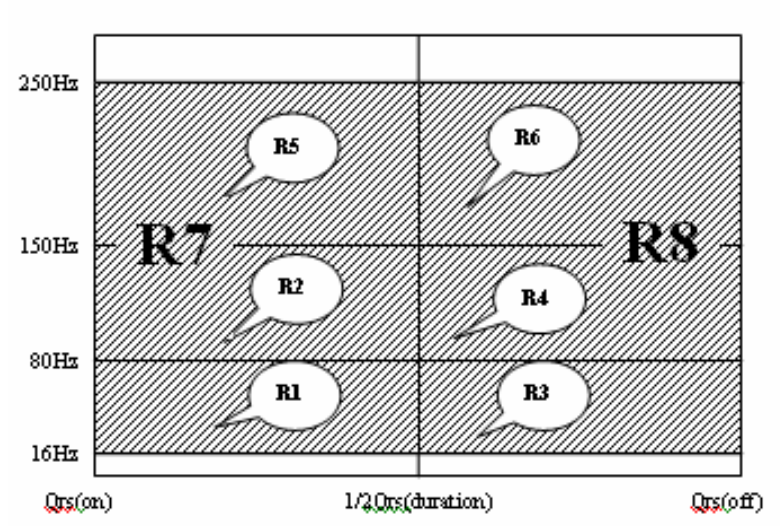

Figure 2: Time-frequency regions of interest.

where $*$ denotes complex conjugate, $a$ the scale (dilation), and $\tau$ the time shift, $1 / \sqrt{a}$ is used for energy normalization and $g_{m}$ is de complex valued wavelet of Morlet expressed as.

$$
g_{m}=e^{j \omega_{o}^{t}} e^{\frac{-t^{2}}{2}}
$$

with $\omega_{\mathrm{o}}=5.3$ this wavelet can be considered approximately analytic and satisfy the following mathematical conditions [11]:

1.- Belong to $\mathbf{L}^{2}(\mathbf{R})$, i.e. be squared integrable (be of finite energy). (see eq-6)

2.- Be analytic $(\mathrm{G}(\omega)=0$ for $\omega<0)$ (approximately)

3.- Be admissible (enable invertibility) (see. eq-7)

We can substitute $t$ and $w$ in eq. (2) with their scaled versions thus obtaining:

$$
g_{m}=e^{j \frac{\omega_{o}}{a} t} e^{\frac{-t^{2}}{2 a^{2}}}
$$

The time-scale representation of (1) can be viewed as a time-frequency one, where the basic analyzing frequency is:

$$
f=\frac{\omega_{0}}{2 \pi a}=\frac{f_{0}}{a} \quad \text { or } \quad a=f_{0} / f
$$

The equation (4) allows setting the frequency band and the calculation of associated scales. Frequencies were fixed according to a logarithmic scale as in (5).

$$
\begin{aligned}
& f i=f 1 B^{(i-1) d} \text { with } d=\frac{\log _{B}\left(\frac{f_{n}}{f_{1}}\right)}{n-1} \\
& \text { for } i=1,2,3, \ldots \ldots \ldots n
\end{aligned}
$$

Once each time-frequency region was set, total energy was calculated as expressed in (6), $w^{2}(\tau, a) / a^{2}$ essentially defines an energy density functions that decomposes the energy across different scales and times [11].

$$
\int_{-\infty}^{+\infty} x(t)^{2} d t=\frac{1}{C_{\psi}} \int_{0}^{\infty} \int_{-\infty}^{\infty} w^{2}(\tau, a) / a^{2} d t d a
$$

where

$$
\begin{aligned}
& C_{\psi} \equiv \int_{0}^{\infty} \frac{|\psi(f)|^{2}}{f} d f, \ldots .0<C_{\psi}<\infty \\
& \psi(f) \equiv \int_{-\infty}^{\infty} \psi(u) e^{-i 2 \pi f u} d u
\end{aligned}
$$

\section{Results}

Total energy was calculated for each region of $\mathrm{R} 1$ to R8 and for each group (RCA, LAD, LCX, and all together). All 12 standard ecg-leads were considered to statistical analysis. RCA group $(\mathrm{N}=23)$ and all arteries $(\mathrm{RCA}+\mathrm{LAD}+\mathrm{LCX})$ presented significant statistical results when comparing HFEC before and during PTCA, in leads II and aVf, as shown at table 1.

\section{Discussion and conclusions}

Results suggest that leads II and aVF are more sensitive to high frequency total energy changes. Lead III was about to reach significant statistical results at the level $p=0.05$. There were not significant results for LAD, LCX groups. First QRS half is not sensitive to highfrequency changes as was expected. High frequency band $80-250 \mathrm{~Hz}$ could be used as a marker for ischemic events during depolarization. "Fingerprint" like sensitive timefrequency area could be overlapped within 16-80-150-250 $\mathrm{Hz}$ bands. Future research should be directed to assess which frequency - domain regions are more sensitive to predict ischemic events during cardiac depolarization. 
Table 1. Total energy in the RCA and RCA+LAD+LCX groups with significant statistical difference.

\begin{tabular}{|c|c|c|c|}
\hline $\begin{array}{l}\text { Time- } \\
\text { Frequency } \\
\text { regions }\end{array}$ & \multicolumn{2}{|c|}{$\begin{array}{l}\text { RCA-Group }(\mathrm{N}=23) \\
\text { High Frequency Energy } \\
\left(\mu \mathrm{V}^{2} / \mathrm{Hz}\right)\end{array}$} & $\begin{array}{l}\text { p-value } \\
\text { Anova }\end{array}$ \\
\hline Region: R3 & $\begin{array}{c}\text { Pre-ptca } \\
(\text { mean+/-SD) }\end{array}$ & $\begin{array}{c}\text { Ptca } \\
\text { (mean+/-SD) }\end{array}$ & \\
\hline Lead II: & $6.18+/-8.08$ & $12.5+/-1.9$ & 0.0410 \\
\hline aVF: & $5.12+/-6.25$ & $11.46+/-2.44$ & 0.0340 \\
\hline \multicolumn{4}{|l|}{ Region: R8 } \\
\hline Lead II: & $2.03+/-2.48$ & $4.00+/-3.80$ & 0.0440 \\
\hline aVF: & $1.59+/ 1.87$ & $3.50+/-3.90$ & 0.0340 \\
\hline \multicolumn{4}{|l|}{ Region: R4 } \\
\hline Lead II: & $2.37+/-2.99$ & $4.79+/-4.62$ & 0.0410 \\
\hline aVF: & $1.89+/-2.27$ & $4.30+/-4.72$ & 0.0320 \\
\hline \multicolumn{4}{|l|}{ Region: R6 } \\
\hline Lead II: & $1.56+/-1.78$ & $2.92+/-2.71$ & 0.0500 \\
\hline \multirow[t]{2}{*}{ aVF: } & $1.18+/-1.32$ & $2.53+/-2.72$ & 0.0370 \\
\hline & \multicolumn{2}{|c|}{$\begin{array}{c}\text { All arteries }(\mathrm{N}=39) \\
\text { High Frequency Energy } \\
\left(\mu \mathrm{V}^{2} / \mathrm{Hz}\right)\end{array}$} & $\begin{array}{l}\text { p-value } \\
\text { Anova }\end{array}$ \\
\hline Region: R3 & $\begin{array}{c}\text { Pre-ptca } \\
(\text { mean+/-SD) }\end{array}$ & $\begin{array}{c}\text { Ptca } \\
\text { (mean+/-SD) }\end{array}$ & \\
\hline Lead II: & $5.43+/-6.82$ & $10.03+/-10.57$ & 0.0250 \\
\hline aVF: & $4.35+/-5.55$ & $8.97+/-10.78$ & 0.0200 \\
\hline \multicolumn{4}{|l|}{ Region: R8 } \\
\hline Lead II: & $1.85+/-2.09$ & $3.29+/-3.33$ & 0.0250 \\
\hline aVF: & $1.43+/-1.71$ & $2.86+/-3.36$ & 0.0210 \\
\hline \multicolumn{4}{|l|}{ Region: R4 } \\
\hline Lead II: & $2.13+/-2.52$ & $3.89+/-4.05$ & 0.0240 \\
\hline aVF: & $1.66+/-2.06$ & $3.42+/-4.10$ & 0.0194 \\
\hline \multicolumn{4}{|l|}{ Region: R6 } \\
\hline Lead II: & $1.47+/-1.51$ & $2.46+/-2.37$ & 0.0305 \\
\hline aVF: & $1.10+/-1.26$ & $2.09+/-2.38$ & 0.0245 \\
\hline
\end{tabular}

Regions: R1, R3: (16-80 Hz); R2, R4: (80-150 Hz); R5, R6:(150-250 Hz) and R7, R8: (80-250 Hz); R1, R2, R5 and R7: $1^{\text {st }}$ QRS half and R3, R4, R6 and R8: $2^{\text {nd }}$ QRS half

\section{Acknowledgements}

This work was supported in part by "Dirección de Investigación, Universidad Simón Bolívar, Sede del Litoral, Venezuela, S1-NCBA-009-00" and "FONACIT, Programa de promoción a la formación de nuevos talentos, Caracas, Venezuela".

\section{References}

[1] Wagner G. Marriot's Electrocardiografía. Marbán, S.L. Madrid, 2002. 10ma Edic. pp. 480.

[2] Wagner NB., DC Sevilla, MW Krucoff, et all. Transient alterations of the QRS complex and ST segment during percutaneous transluminal ballon angioplasty of the left anterior descending artery. Am J Cardiol 1988; 62:10381042.

[3] Abboud S, Cohen R, Selwyn A, Ganz Peter, Sadeh D, Friedman P. Detection of transient myocardial ischemia by computer analysis of standard and signal-averaged highfrequency electrocardiograms in patients undergoing percutaneos trasluminal coronary angioplasty. Circulation 1987; 76(3):585-596.

[4] Petterson J, Lander P, Pahlm O, Sornmo L, Warren SG, Wagner GS. Electrocardiographic changes during prolonged coronary artery occlusion in man: comparison of standard and high-frequency recordings. Clinical Phisiology 1998; 18(3): 179-86

[5] Morlet D, Peyrin F, Desseigne P, Touboul P ,P Rubel, TimeScale Analisys of High Resolution Signal-Averaged Surface ECG using Wavelet Transformation. Computers in Cardiology. 1991; pp. 393-396.

[6] Morlet D, Couderc J, Touboul P, Rubel P. Wavelets analysis of high-resolution ECGs in post-infarction patients: role of the basic wavelet and of the analyzed lead. Int J Bio-Medical C; 39: 311-325. April 1995.

[7] Gramatikov B, Brinker J,Yi-chun S,Thakor N. Wavelet analysis and time-frequency distributions of the body surface ECG before and after angioplasty. Comp Meth and Programs in Biomedicine 2000; 62:87-98.

[8] Mor-Aviv V, Shargorodsky B, Abboud Shimon, Laniado S, Akselrod. Effects of coronary occlusion on high-frequency content of the epicardial electrogram and body surface electrocardiogram. Circ. July 1987; 76(1): 237-243.

[9] Ringborn M, Pahlm O, Wagner G, Warren S, Petterson J. The absence of high-frequency QRS changes in the presence of standard electrocardiographic QRS changes of old myocardial infarction. Am Heart J 2001; 141(4): 573579.

[10] Selvester RH., Wagner GS, Hindman NB. The development and application of the Selvester QRS scoring system for estimating myocardial infarct size. Arch Intern Med 1985; 145:1877-81.

[11] Percival D, Walden A. Wavelet Methods for Time Series Analysis. Cambridge University Press. 1ra. Ed. New York, 2000. pp. 594.

Address for corresponcence

Noel Camilo Castro,

Dpto. Electrónica y Circuitos Edif. FE-1, Universidad Simón Bolívar, Sartenejas, Caracas-Venezuela. C.P. 1080. noelcastro69@hotmail.com 